\title{
Welcher Marker ist am präzisesten?
}

Fragestellung: Welcher Biomarker oder kognitive Marker sagt die Entwicklung der Alzheimer-Demenz bei Patienten mit leichter kognitiver Störung am genauesten voraus?

Hintergrund: In den letzten Jahren wurden mehrere vielversprechende Marker der Alzheimer-Erkrankung etabliert, zum Beispiel regionale Hirnvolumina, Liquorproteine und kognitive Maße. Das Ziel dieser Studie war es, diese Marker sowie die individuellen Risikofaktoren wie Alter, Ausbildung, und ApoEStatus bezüglich ihrer Fähigkeit, die Entwicklung der Alzheimer-Demenz vorherzusagen, zu vergleichen.

Patienten und Methodik: Die longitudinale Studie wertete die Daten der öffentlich verfügbaren multizentrischen Datenbank

Gomar JJ, Bobes-Bascaran MT, Conejero-Goldberg C et al, Alzheimer's Disease Neuroimaging Initiative. Utility of combinations of biomarkers, cognitive markers, and risk factors to predict conversion from mild cognitive impairment to Alzheimer disease in patients in the Alzheimer's disease neuroimaging initiative. Arch Gen Psychiatry 2011; 68: 961 -9
Alzheimer's disease neuroimaging initiative (ADNI) aus [1]. Eingeschlossen wurden alle Patienten mit leichter kognitiver Störung (mild cognitive impairement, MCI), die eine Baseline- und mindestens eine Follow-up-Untersuchung bekamen. Abhängig davon, ob die Patienten innerhalb von zwei Jahren nach der BaselineUntersuchung die AlzheimerDemenz entwickelten, wurden zwei Gruppen gebildet: MCI-Konverter $(\mathrm{n}=116)$ und MCINichtkonverter $(\mathrm{n}=204)$. Analysiert wurde, welches Baseline$\mathrm{Maß}$ die Konversion innerhalb von zwei Jahren am genauesten prädiziert. Darüber hinaus wurde geprüft, ob das Ausmaß der Veränderung über die Zeit (Baseline zu einem oder zwei Jahren) die Konversion voraussagen kann.

Ergebnisse: Der ApoE-Status war der einzige signifikante Konversionsprädiktor unter den demografischen und individuellen Charakteristika. Bei den kognitiven Markern waren Auditory verbal learning Test list recall at $30 \mathrm{~min}$, logic memory delayed recall, ADAS memory, Clock drawing test und TMT A signifikant, bei den volumetrischen Biomarkern die Volumina des linken Hippokampus und des linken medialen Temporallappens.

Wurden nur die signifikanten Prädiktoren in ein Modell eingeschlossen, blieben drei Variablen übrig: logic memory delayed recall, Volumen des linken medialen Temporallappens und der Auditory verbal learning Test. Bei Bildung und Betrachtung von Differenzwerten (Baseline zu einem Jahr) sagten nur der Functional Assessment Questionnaire und TMT B die Konversion signifikant voraus.

Schlussfolgerungen: Kognitive Marker sind robustere Prädiktoren der Konversion als die meisten Biomarker. Die Konversion wird weniger durch neurobiologische Faktoren der Erkrankung als vielmehr durch den akuten Abfall funktioneller Fähigkeiten und einer Verschlechterung der Exekutivfunktion getrieben.

\section{-Kommentar von Dr. Igor Yakushev}

\section{Neuropsychologische Testung ist ein effizientes Diagnoseinstrument}

Die generelle Überlegenheit der kognitiven Marker stützt die Ansicht, dass ein relativ leicht eruierbares und günstiges Instrument wie die neuropsychologische Testung hoch effizient im Rahmen der Demenzdiagnostik sein kann. Die Konsequenzen der Studie für den klinischen Alltag liegen auf der Hand: Eine neuropsychologische Testung inklusive eines Tests zur Messung des verzögerten Abrufs sowie eine MR-Bildgebung, die im deutschsprachigen Raum ohnehin zur Standarddiagnostik der Demenz gehören, reichen in einem unkomplizierten Fall aus, um die wichtigsten prognostischen Informationen zu gewinnen. Da das (linke) hippokampale Volumen im Vergleich zum (linken) Volumen des medialen Temporallappens nur eine etwas niedrigere prognostische Validität aufwies, wäre dieses aufgrund einer leichteren qualitativen und quantitativen Messbarkeit eventuell zu bevorzugen. Die Tatsache, dass das Ausmaß der regressiven Veränderung der funktionellen Fähigkeiten - und nicht der Biomarker - entscheidend für die Konversion und die Diagnosestellung der Demenz war, spiegelt im Wesentlichen die geläufigen und in der Studie verwendeten Definitionen der (wahrscheinlichen) Alzheimer-Erkrankung und $\mathrm{MCl}$, das heißt Vorliegen versus Fehlen der funk- tionell relevanten Einschränkung im Alltag, wider. Daraus folgt, dass diese Ergebnisse vor dem Hintergrund der verwendeten diagnostischen Kriterien zu deuten sind. Eine Verwendung anderer, „Biomarker-plastischer" Kriterien der Alzheimer-Erkrankung [2] könnte durchaus zu anderen Ergebnissen führen.
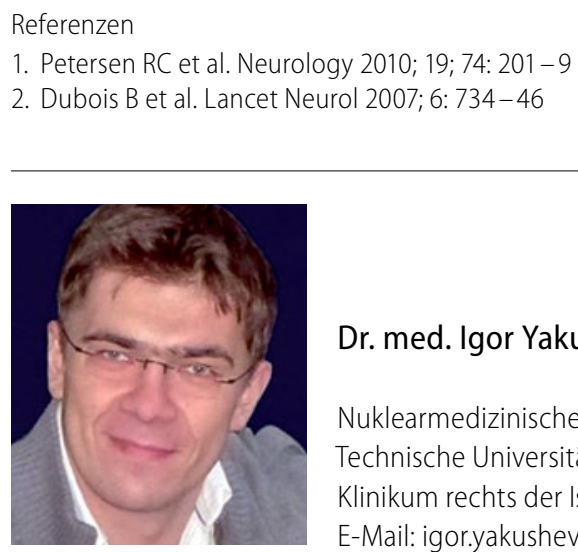

Dr. med. Igor Yakushev, München

Nuklearmedizinische Klinik und Poliklinik, Technische Universität München, Klinikum rechts der Isar E-Mail: igor.yakushev@|rz.tum.de 